\title{
Photosensitizing properties of supramolecular systems based on chlorin $\mathrm{e}_{6}$
}

\author{
Inna V. Klimenko ${ }^{1^{*}}$, and Anton V. Lobanov ${ }^{2}$ \\ ${ }^{1}$ Emanuel Institute of Biochemical Physics of Russian Academy of Sciences, 4 Kosygin str., Moscow 119334, Russia \\ ${ }^{2}$ Semenov Institute of Chemical Physics of Russian Academy of Sciences, 4 Kosygin str., Moscow 119334, Russia \\ *e-mail: inna@deom.chph.ras.ru
}

\begin{abstract}
The novel supramolecular systems based on chlorine e6 $\left(\mathrm{Ce}_{6}\right)$ are presented, and their optical absorption and fluorescence have been investigated. The influence of different excipients as poly-N-vinylpyrrolidone (PVP), polyethyleneglycol (PEG), bovine serum albumin (BSA), chitosan, Triton X-100 (TX-100) on spectral characteristics of these systems has been studied. The obtained spectral-fluorescence characteristics of $\mathrm{Ce}_{6}-\mathrm{PVP}, \mathrm{Ce}_{6}-\mathrm{PEG}, \mathrm{Ce}_{6}-\mathrm{BSA}, \mathrm{Ce}_{6}-\mathrm{TX}-100$ indicate disaggregation of chlorine $\mathrm{e}_{6}$ molecules and their consecutive interaction with excipients in solutions and formation of molecular associates and molecular complexes. The system $\mathrm{Ce}_{6}$-chitosan is characterized by aggregation of pigments in solution that reduces photochemical activity of a photosensitizer. The fluorescence quantum yield $\varphi_{\mathrm{k}}$ of mentioned above supramolecular systems has been calculated. The results can be useful during the new method of controlled aggregation of photosensitizers as a part of supramolecular complexes development as well as for the purposes of new medicines of predictable photodynamic activity creation. (C) 2016 Journal of Biomedical Photonics \& Engineering.
\end{abstract}

Keywords: chlorin e6, photodynamic therapy, supramolecular systems, spectral characteristics, optical absorption, fluorescence

Paper \#3122 received 2016.11.20; accepted for publication 2016.12.29; published online 2016.12.31. doi: 10.18287/JBPE16.02.040310. [Saratov Fall Meeting 2016 Special Issue].

\section{References}

1. A. B. Uzdensky, Cellular and molecular mechanisms of photodynamic therapy, Science, SPb, Russia (2010).

2. A. L. Akopov, N. V. Kazakov, A. A. Rusanov, and A. Karlson, "The mechanisms of photodynamic action for treating of cancer patients," Photodynamic therapy and photodiagnostics 4(2), 9-16 (2015) [in Russian].

3. E. V. Pinyaskina, "Photodynamic processes in cells. Primary mechanisms," Bulletin of DSPU 1, 60-67 (2010) [in Russian].

4. E. Zenkevich, E. Sagun, V. Knyukshto, A. Shulga, A. Mironov, O. Efremova, R. Bonnett, S. P. Songca, and M. Kassem, "Photophysical and photochemical properties of potential porphyrin and chlorin photosensitizers for PDT," J. of Photochemistry and Photobiology B: Biology 33(2) 171-180 (1996).

5. M. V. Parkhats, V. A. Galievsky, A. S. Stashevsky, T. V. Trukhacheva, and B. M. Dzhagarov, "Dynamics and efficiency of the photosensitized singlet oxygen formation by chlorin e6: the effects of the solution $\mathrm{pH}$ and polyvinylpyrrolidone," Optics and Spectroscopy 107(6), 974-980 (2009).

6. C. T. H. Yen, G. V. Ramenskaya, and N. A. Oborotova, "Chlorin derivatives in cancer photodynamic therapy," Rus. J. of Biotherapy 8(4), 99-104 (2009) [in Russian].

7. M. V. Parkhats, V. N. Knyukshto, H. A. Isakau, P. T. Petrov, and B. M. Dzhagarov, "Photophysical properties of photosensitizer chlorin e6 incorporated into polyvinylpyrrolidone," Proc. SPIE 6727, 67272L (2007).

8. T. E. Zorina, I. V. Yankovsky, I. E. Kravchenko, T. V. Shman, M. V. Belevtsev, and V. P. Zorin, "Evaluation of Phototoxicity and cytotoxicity for chlorin e6 ester derivatives and their liposomal forms," Biophysics 60(5), 759-766 (2015).

9. E. A. Genina, Biophotonics methods: Phototherapy, New Wind, Saratov, Russia (2012). 
10. H. A. Isakau, M. V. Parkhats, V. N. Knyukshto, B. M. Dzhagarov, E. P. Petrov, and P. T. Petrov, “Toward understanding the high PDT efficacy of chlorin e6-polyvinylpyrrolidone formulations: Photophysical and molecular aspects of photosensitizer-polymer interaction in vitro," J. of Photochem. and Photobiol. B: Biology 92(3), 165-174 (2008).

11. A. K. Visheratina, A. O. Orlova, V. G. Maslov, A. V. Fedorov, and A. V. Baranov, "Optical properties of conjugates of $\mathrm{CdSe} / \mathrm{ZnS}$ quantum dots and chlorin e6 in aqueous solution," J. of Opt. Tech. 82(11), 738-742 (2015).

12. S. L. Fiedaruk, T. V. Trukhacheva, S. N. Sokolov, K. A. Frolenko, and V. P. Kheidorov, "The study of spectral properties of dimethylester of chlorin e6 in the presence of polyvinilpyrrolidone," Vestnik farmacii 3(65), 8288 (2014) [in Russian].

13. V. Yu. Plavskii, V. A. Mostovnikov, G. R. Mostovnikova, A. I. Tret'yakova, and A. V. Mikulich, "Spectralluminescent properties of chlorin e6 and malate dehydrogenase complexes," J. of Appl. Spectroscopy 71(6), 818-828 (2004).

14. G. P. Gurinovich, A. I. Sevchenko, and K. N. Solov'ev, Spectroscopy of a chlorophyll and related compounds, Science and technology, Minsk, USSR (1978).

15. H. Podbielska, A. Ulatowska-Jarza, G. Muller, I. Holowacz, J. Bauer, and U. Bindig, "Silica sol-gel matrix doped with Photolon molecules for sensing and medical therapy purposes," Biomolecular Engineering 24(5), 425-433 (2007).

16. A. A. Krasnovsky, "Photodynamic action and singlet oxygen," Biophysics 49(2), 289-306 (2004) [in Russian].

17. G. V. Golovina, V. A. Ol'shevskaya, V. V. Vargin, V. A. Kuzmin, and A. B. Shevelev, "Solubility of porphyrine derivatives in vivo affected by aggregate formation and complexes formation with serum albumin," Health of the population and habitat 12, 41-43 (2013) [in Russian].

18. N. H. Karapetyan, and V. N. Madakyan, "The Interaction of new pyridylporphyrins with bovine serum albumin,” Russ. J. of Boiorganic Chemistry 30(2), 172-177 (2004) [in Russian].

\section{Introduction}

Photodynamic therapy (PDT) is clinically used as a nonsurgical treatment in oncology, ophthalmology, dermatovenerology, and others, for various diseases, for example, cancers and bacterial cells. It is based on the use of a special chemical agent - photosensitizer (PS), which is accumulated in pathological target cells and initiated by laser light of an appropriate wavelength, corresponding photosensitizer absorption maxima. As a result, photochemical reactions between PS and oxygen, leading to the formation of cytotoxic agents in tumor tissue, damaging a tumor, take place. At this, photosensitizer transits into exited state, and PS molecule undergoes or oxidation-reduction reactions with electrons or protons transfer and formation of intermediate products - the radicals, further interacting with oxygen, or transfers excess of energy to the molecule of oxygen transiting at the same time into the excited singlet state [1].

In real biological systems both mechanisms can take place. Their relative contribution to bioprocesses depends on the PS identity, a concentration and a nature of the oxidized reagents, a concentration of oxygen and some other factors.

Nowadays most of the clinically used and experimentally tested PSs belong to the class of porphyrines or their reduced analogues, chlorins [2]. In biosystems in the presence of oxygen they usually enter photochemical reactions of the II type as the first triplet state energy of porphyrines $(200 \mathrm{~kJ} / \mathrm{mol})$ is sufficient for ${ }^{1} \mathrm{O}_{2}(95 \mathrm{~kJ} / \mathrm{mol})$ development [3].

Chlorin $\mathrm{e}_{6}\left(\mathrm{Ce}_{6}\right)$ belongs to the class of the plant porphyrines purchasing from chlorophyll a. It is anticancer immune response modifier, activating the system of cell-mediated immunity and it possesses the following spectral and energy characteristics: the maximum of absorption is in the region of $660 \pm 5 \mathrm{~nm}$ that corresponds to the region of the greatest optical transparency for biological tissue, and a quantum yield of singlet oxygen reaches $0.7[4,5]$. Three $\mathrm{COOH}$ groups provide the $\mathrm{Ce}_{6}$ molecule hydrophilic properties, effective transport and accumulation in tumour tissue. High photodynamic activity when using small doses and low toxicity are also characteristics of $\mathrm{Ce}_{6}$. Moreover, the availability of raw materials (microalgae Spirulina platensis or leaves of a nettle) plays a large role in the development of compounds based on chlorin.

Such "second-generation" photosensitizers as Radachlorine, Photolon, Potoditazin and others of chlorin alignment are in medical application in Russia. Various physical, chemical and pharmacological properties, and also various commercial costs are the characteristic of all these medicines [6]. However, these PSs have the high degree of phototoxicity connected with a high quantum yield of the singlet oxygen which is formed under the light influence when carrying out PDT. Besides, $\mathrm{Ce}_{6}$, as a substance, has a number of shortcomings limiting its broad consumption, for example, a small period of storage and a loss of optical properties, elimination within 48 hours or more. It should be noted that chlorin molecules also tend to form aggregates in water solutions that leads to considerable decrease in photodynamic activity $[5,7]$. Besides, the aggregation processes of the porphyrine pigments take place in polar solvents because of hydrophobic and $\pi-\pi$ interactions of tetrapyrrol structures [8]. To avoid these 
it's reasonable to develop and study the new effective systems with the optimum photophysical and pharmacological properties.

In this work the novel supramolecular systems based on chlorin $\mathrm{e}_{6}$ have been obtained and the influence of various excipients on spectral characteristic of these systems as well as the influence of intermolecular interactions on aggregation behavior of chlorin $\mathrm{e}_{6}$ in solutions have been studied.

\section{Materials and methods}

$\mathrm{Ce}_{6}$ (Fig. 1) was kindly provided by the colleagues from Moscow Technological University (Preobrazhensky Department of chemistry and technology of biologically active compounds). Basic $\mathrm{Ce}_{6}$ solutions with the concentration of $2.0 \cdot 10^{-3} \mathrm{~mol} / \mathrm{l}$ were prepared by dissolution of $\mathrm{Ce}_{6}$ dry sample weight in $20 \mathrm{ml}$ of dimethylformamide (DMF) double-distilled. The received concentration was specified on electron absorption spectrum. Solutions stored in the dark at +4 ${ }^{0} \mathrm{C}$. Purity and identity of $\mathrm{Ce}_{6}$ were confirmed with the MALDI - mass spectrometry method on the Thermo Scientific DSQ II single quadrupole mass spectrometer (Thermo Scientific, USA)

The choice of water-soluble polymers (Fig. 1) is explained by the fact that they are the most widespread biologically-compatible substances which are the part of physiological solutions applied as a part of pharmacological drug. Supramolecular systems based on $\mathrm{Ce}_{6}\left(2 \cdot 10^{-5} \mathrm{M}\right)$ in various excipients were studied: poly-N-vinylpyrrolidone (PVP) - $\mathrm{Ce}_{6}$,

polyethyleneglycol (PEG) - $\mathrm{Ce}_{6}$, Triton X-100 (TX-100) - $\mathrm{Ce}_{6}$, bovine serum albumin (BSA) - $\mathrm{Ce}_{6}$, chitosan $\mathrm{Ce}_{6}$. Water solutions of Triton X-100 micelles (TX-100, $\mathrm{C}_{34} \mathrm{H}_{62} \mathrm{O}_{11}$, LAB-SCAN analytical sciences, Poland) of concentration $2.5 \cdot 10^{-4} \mathrm{~mol} / \mathrm{l}$, and $2 \%$ (weight) solutions of poly-N-vinylpyrrolidone (PVP, $(\mathrm{M}=26500)$, SigmaAldrich, Germany) and polyethyleneglycol (PEG, $(\mathrm{M}=1000)$ MERCK, Germany) were prepared separately.

Absorption spectra $(200-900 \mathrm{~nm})$ were recorded with TU-1901 UV-Vis spectrophotometer from Beijing Purkinje General Instruments Co Ltd. Poorly resolved spectra were analyzed by decomposing the spectra into their Gaussian constituents.

The fluorescence spectra in the range of 550-800 nm were recorded with Fluorat-02-Panorama spectrofluorimeter (Lumex, Russia). The excitation wavelength was $410 \mathrm{~nm}$. All measurements were conducted at $20{ }^{\circ} \mathrm{C}$ in standard $\mathrm{K} 10$ quartz cuvettes with optical path lengths of $1 \mathrm{~cm}$. Registration of singlet oxygen was carried out by the method of "chemical traps"; 1,3-diphenylisobenzofuran was used as a "trap".

\section{Results and discussion}

Obtained in this experiment optical absorption spectrum of $\mathrm{Ce}_{6}$ aqueous solution practically corresponds to the data of $\mathrm{Ce}_{6}$ optical absorption spectrum presented in [913]. It is characterized by a strong Soret band with a peak at $\lambda=403 \mathrm{~nm}$, typical for all porphyrines [14] and weaker Q-band in the region of 450-750 nm, which include a plateau at $\lambda=450-615 \mathrm{~nm}$ with small peaks at 504,533 and $599 \mathrm{~nm}$, and also high-intensity peak at $\lambda=653 \mathrm{~nm}$. It should be noted that the penetration depth of light is limited by absorption and scattering in tissue [15]. Besides, the $\mathrm{Ce}_{6}$ line shift to the long-wavelength region increases tissue permeability for visible light and at the same time reduces light absorption by blood pigment at 500-600 nm. All of these play an essential role for increase of PDT effectiveness [9].

(a)

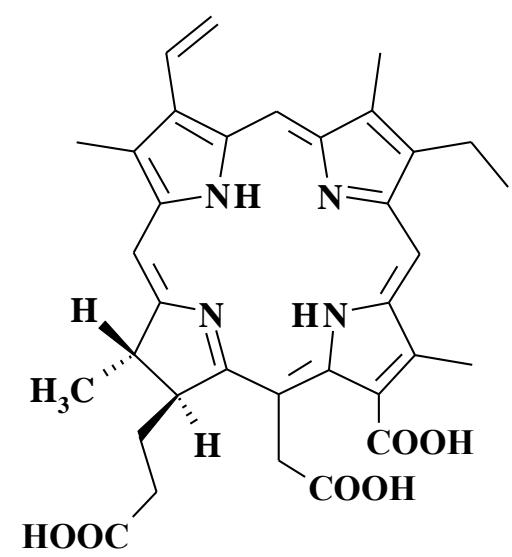<smiles>CC(C)(C)C(N1CCCC1=O)C(C)(C)C</smiles>

(b)

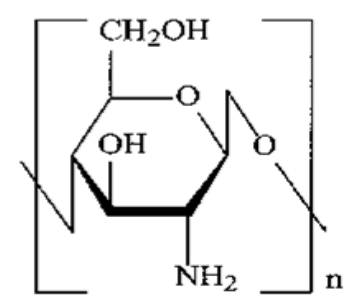

(e)

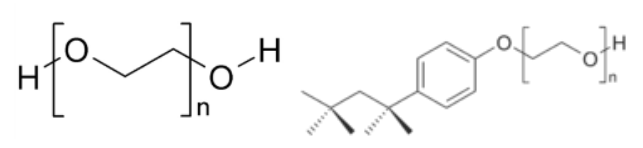

(c)

(d)
Fig. 1 Structures of chlorin $\mathrm{e}_{6}$ (a), poly-Nvinylpyrrolidone (b), polyethyleneglycol (c), Triton X100 (d), chitosan (e), bovine serum albumin (f).

When studying spectral properties of all presented supramolecular systems based on $\mathrm{Ce}_{6}$, the hypsochromic shift in the field of Soret peak wasn't revealed (Fig. 2). According to the ideas of spectral shifts in biopolymers [12], the hypsochromic shift is connected with the transition of chromophore to more polar environment, and in our case, polar aqueous solvent. Absence of hypsochromic shift in the field of Soret peak testifies the identical hydrophobic behavior of all supramolecular systems, similar to hydrophobic behavior of $\mathrm{Ce}_{6}$.

As illustrated in the Fig. 2, a small shift of Soret band to the long-wavelength region of optical 
absorption spectra of $\mathrm{Ce}_{6}-\mathrm{PVP}$ and $\mathrm{Ce}_{6}$-BSA systems by 2 and 5 nanometers, respectively, in comparison to one of $\mathrm{Ce}_{6}$, is observed. Besides, some broadening of spectral band can be seen for the $\mathrm{Ce}_{6}-$ PVP system. The system of $\mathrm{Ce}_{6}-$ PEG shows a sharp splitting of Soret band with a form of Gaussian distribution and broadening by $\Delta \lambda^{1 / 2}$ Soret $=13 \%$. On the basis of absorbency fall in the region of Soret band for all systems and according to [16] one can say of single oxygen formation in all presented supramolecular systems. Besides, hypsochromic effect can be connected with the strengthening of intermolecular interactions (including interactions between the chromophores which are in more ordered form in comparison with the free state), and, as a result, the formation of molecular complexes takes place. This statement is consistent with the conclusions made by the authors of $[5,12]$, showed that, upon interactions, $\mathrm{Ce}_{6}$ forms complexes with PVP. Moreover, the results of $[17,18]$ devoted to the investigation of porphyrines-serum albumin systems also confirm our hypotheses.

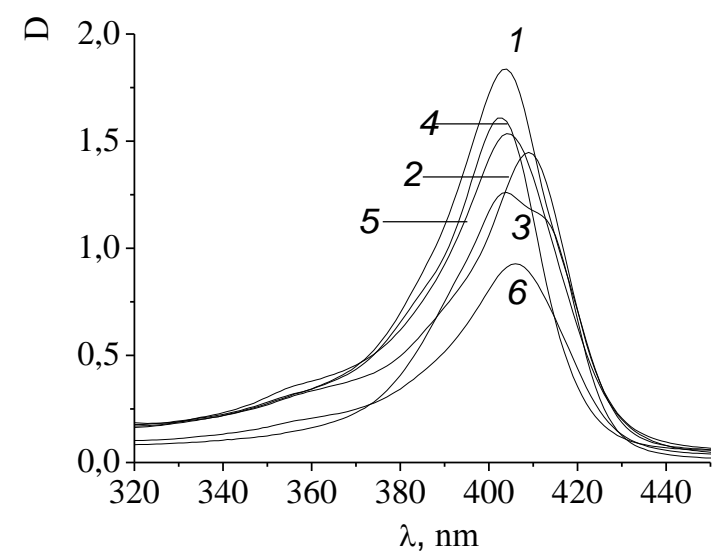

Fig. 2 Absorption spectra of supramolecular systems based on $\mathrm{Ce}_{6}\left(2 \cdot 10^{-5} \mathrm{M}\right)$ in the region of the Soret peak: $1-\mathrm{Ce}_{6}$ in aqueous solution, $2-\mathrm{Ce}_{6}-\mathrm{BSA}, 3-\mathrm{Ce}_{6}-\mathrm{PEG}$, 4 - $\mathrm{Ce}_{6}$-chitosan, 5 - $\mathrm{Ce}_{6}$-TX-100, 6 - $\mathrm{Ce}_{6}$-PVP.

Optical absorption spectra (Q-band region) of supramolecular systems based on $\mathrm{Ce}_{6}\left(2 \cdot 10^{-5} \mathrm{M}\right)$ in various excipients are shown in Fig. 3. When comparing the received data with the data for $\mathrm{Ce}_{6}$ aqueous solution, we have revealed that in a case of chitosan, PVP, TX100 and $\mathrm{BSA}$ addition to $\mathrm{Ce}_{6}$ the maximum of longwavelength Q-band absorption $(\lambda=653 \mathrm{~nm})$ undergoes bathochromic shift $(1 \mathrm{~nm}, 10 \mathrm{~nm}, 12 \mathrm{~nm}$ and $13 \mathrm{~nm}$, correspondingly), whereas in the $\mathrm{Ce}_{6}-\mathrm{PEG}$ system the hypsochromic shift of the Q-band maximum $(14 \mathrm{~nm})$ is observed. As a result, the maximum of Q-band absorption changes in row: PEG (639 nanometers) < chitosan $(654 \mathrm{~nm})<$ PVP $(663 \mathrm{~nm})<\mathrm{TX}-100(665 \mathrm{~nm})$ $<$ BSA $(666 \mathrm{~nm})$. Thus, absorption maximum shift to the red region of spectrum demonstrates that when performing photodynamic therapy with the systems having PVP, TX-100 and BSA as excipients there is a high probability of penetrating light absorption and big prospects for invasion these systems through tissues in comparison with $\mathrm{Ce}_{6}$.

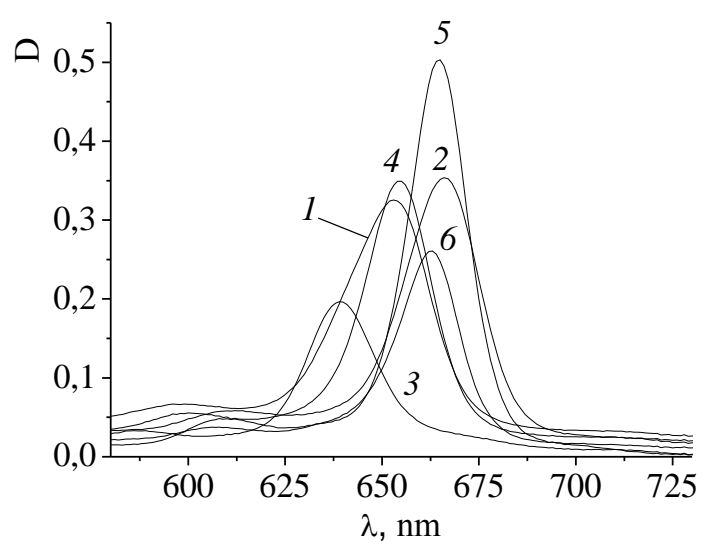

Fig. 3 Absorption spectra of supramolecular systems based on $\mathrm{Ce}_{6}\left(2 \cdot 10^{-5} \mathrm{M}\right)$ in the region of the Q-band: 1 $\mathrm{Ce}_{6}$ in aqueous solution, $2-\mathrm{Ce}_{6}-\mathrm{BSA}, 3-\mathrm{Ce}_{6}-\mathrm{PEG}, 4$ $\mathrm{Ce}_{6}$-chitosan, 5 - $\mathrm{Ce}_{6}$-TX-100, 6 - $\mathrm{Ce}_{6}$-PVP.

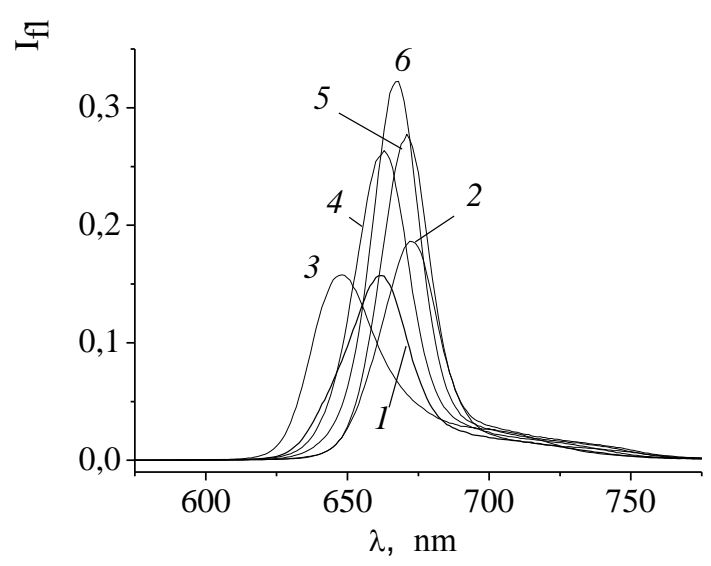

Fig. 4 Fluorescence spectra of supramolecular systems based on $\mathrm{Ce}_{6}\left(2 \cdot 10^{-5} \mathrm{M}\right): 1-\mathrm{Ce}_{6}$ in aqueous solution, 2 $\mathrm{Ce}_{6}$-BSA, $3-\mathrm{Ce}_{6}-\mathrm{PEG}, 4-\mathrm{Ce}_{6}$-chitosan, $5-\mathrm{Ce}_{6}-\mathrm{TX}-$ $100,6-\mathrm{Ce}_{6}-\mathrm{PVP}$.

The spectral changes in the Q-band region of absorption spectrum connected with the bathochromic shift of a long - wavelength absorption band of a sensitizer and essential reduction of half-width of a line also demonstrate disaggregation of $\mathrm{Ce}_{6}$ molecules, their interaction with excipients in solutions and formation of a molecular complex. The appearance of a porphyrine molecular complex with TX-100, BSA and PVP absorption band at $615 \mathrm{~nm}$ (Fig. 3) is the confirmation of this assumption. Moreover, the disappearance of absorption in the region of $680-700 \mathrm{~nm}$ for $\mathrm{Ce}_{6}-$ PEG system, specific for porphyrine aggregates, is also the confirmation of the porphyrine aggregates destruction and transition of a PS to molecular associates, and at a later stage, possibly, molecular complexes [17]. 
The specific characteristic of fluorescence spectra of all supramolecular systems is their overlap with Q-band of absorption spectra (Fig. 4), that allows considering the formed molecular associates and complexes as donors of photoexitation energy for $\mathrm{Ce}_{6}$ molecules. As can be seen from the Fig. 4, the addition of all studied excipients into $\mathrm{Ce}_{6}$ aqueous solution leads to the increase of fluorescence intensity of $\mathrm{Ce}_{6}$. The greatest increase in fluorescence intensity is observed in the presence of PVP. Moreover, the determined by a relative method [13] (the free form of the dye in the absence of the enzyme was as a standart with $\varphi_{0}=0.15$ ) quantum yield $\varphi_{\mathrm{k}}$ of the fluorescence is depends on the excipients, changing from 0.12 for TX-100 and 0.13 for chitosan to 0.16, in PEG and PVP. Such supramolecular compound as $\mathrm{Ce}_{6}$-BSA shows the value of the fluorescence quantum yield as 0.15 equal to the fluorescence quantum yield for $\mathrm{Ce}_{6}$.

Some increase in $\varphi_{\mathrm{k}}$ value for $\mathrm{Ce}_{6}-\mathrm{PEG}$ and $\mathrm{Ce}_{6}-$ $\mathrm{PVP}$ in comparison with $\varphi_{\mathrm{k}}$ value for $\mathrm{Ce}_{6}$ aqueous solution confirms the data of optical absorption spectra and demonstrates consecutive destruction of $\mathrm{Ce}_{6}$ aggregates and a complex formation. It should be noted that aggregates degradation rate in the aqueous environments is various in the presence of different excipients. Probably, the calculated for $\mathrm{Ce}_{6}$-BSA system value of a quantum yield, equal to the same value for $\mathrm{Ce}_{6}$, can be explained by this. Low $\varphi_{\mathrm{k}}$ value for $\mathrm{Ce}_{6}-$ TX-100, possibly, is connected with the partial aggregation of a pigment which is due to low solubility and the formation of another type complex characterized by a low fluorescence quantum yield. As far as fluorescent characteristics of the $\mathrm{Ce}_{6}-$ chitosan system, it is seen that they remain almost invariable in comparison with similar characteristics of $\mathrm{Ce}_{6}$ aqueous solution, and some decrease in $\varphi_{\mathrm{k}}$ is explained, perhaps, by aggregation of dye in solution that reduces photochemical activity of a PS.

With the help of the method of "chemical traps" the registration of singlet oxygen was carried out. From this, one can assume that as a part of the supramolecular complexes, $\mathrm{Ce}_{6}$ is an effective photosensitizer of singlet oxygen generation with a quantum yield $0.53\left(\mathrm{Ce}_{6}{ }^{-}\right.$ PVP), 0.50 ( $\left.\mathrm{Ce}_{6}-\mathrm{PEG}\right), 0.62\left(\mathrm{Ce}_{6}-\mathrm{TX}-100\right)$ and 0.56 $\left(\mathrm{Ce}_{6}-\mathrm{BSA}\right)$.

\section{Conclusion}

To sum up, the conducted study of spectral and fluorescent characteristics of various supramolecular systems on the basis of chlorin $\mathrm{e}_{6}$ allows us to conclude promising method of controlled aggregation of photosensitizers in the composition of supramolecular complexes to create medicines of predictable photodynamic activity. The obtained Q-band absorption maxima are in a row: PEG (639 nanometers) < chitosan $(654 \mathrm{~nm})<\operatorname{PVP}(663 \mathrm{~nm})<$ TX-100 $(665 \mathrm{~nm})<$ BSA $(666 \mathrm{~nm})$, which clearly demonstrates the disaggregation of $\mathrm{Ce}_{6}$ molecules in systems with TX100, BSA and PVP. The appearance of a porphyrine molecular complex absorption band at $615 \mathrm{~nm}$ for $\mathrm{Ce}_{6-}$ TX-100, $\mathrm{Ce}_{6}-\mathrm{BSA}$ and $\mathrm{Ce}_{6}-\mathrm{PVP}$ systems also shows the $\mathrm{Ce}_{6}$ molecules disaggregation. As far as $\mathrm{Ce}_{6}-\mathrm{PEG}$ system, the absence of absorption in the region of 680$700 \mathrm{~nm}$ testifies the porphyrine aggregates destruction. In addition, the obtained from fluorescence spectra value of $\varphi_{\mathrm{k}}=0.16$ for $\mathrm{Ce}_{6}-\mathrm{PEG}$ is consistent with the absorption data and confirms the appearance of disaggregated $\mathrm{Ce}_{6}$ molecules fraction. A quantum yield of singlet oxygen generation falls in the range from 0.5 for PEG up to 0,62 for TX-100. According to [5], values received are close or exceed the corresponding value for chlorin $\mathrm{e}_{6}$ in the aqueous solution $(0.5 \pm 0.05$ at the medium of $\mathrm{pH} 6.3$ ), which confirms the assumption on the disaggregating action of excipients.

Though, the presented systems call for the further investigations, spectral and fluorescence data clearly demonstrate the absence of aggregation in such systems as $\mathrm{Ce}_{6}-\mathrm{PVP}, \mathrm{Ce}_{6}-\mathrm{PEG}, \mathrm{Ce}_{6}-\mathrm{BSA}$ and $\mathrm{Ce}_{6}-\mathrm{TX}-100$ and the prospects of use of these systems for diagnostics (and, as possible, therapy) are evident. 